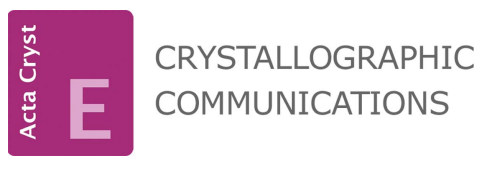

ISSN 2056-9890

Received 16 March 2020

Accepted 16 April 2020

Edited by J. T. Mague, Tulane University, USA

Keywords: crystal structure; 2-phenyl-1-Hphenanthro $[9,10-d]$ imidazole; hydrogen

bonding; $\pi-\pi$ interactions.

CCDC reference: 1997348

Supporting information: this article has supporting information at journals.iucr.org/e

\section{Crystal structure and Hirshfeld surface analysis of 2-phenyl-1H-phenanthro[9,10-d]imidazol-3-ium benzoate}

\author{
Ruby Ahmed, ${ }^{\text {a }}$ Onur Erman Doğan, ${ }^{\text {b }}$ Farman Ali, ${ }^{a}$ Musheer Ahmad, ${ }^{a}$ Adeeba \\ Ahmed, ${ }^{a}$ Necmi Dege $^{c_{*}}$ and Irina A. Golenia ${ }^{d_{*}}$
}

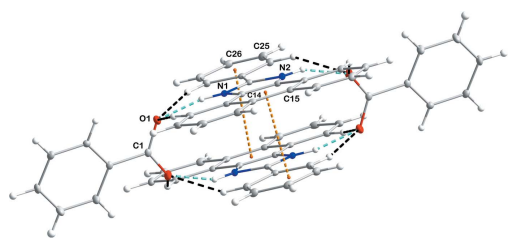

OPEN $\partial$ ACCESS
aDepartment of Applied Chemistry, ZHCET, Aligarh Muslim University, Aligarh, 202002, UP, India, ${ }^{\mathbf{b} O n d o k u z ~ M a y ı s ~}$ University, Faculty of Arts and Sciences, Department of Chemistry, 55139 Samsun, Turkey, ' Ondokuz Mayıs University, Faculty of Arts and Sciences, Department of Physics, 55139 Samsun, Turkey, and d Department of Chemistry, Taras Shecchenko National University of Kyiv, 64, Vladimirska Str., Kiev 01601, Ukraine. *Correspondence e-mail: necmid@omu.edu.tr, igolenya@ua.fm

In the title compound, $\mathrm{C}_{21} \mathrm{H}_{15} \mathrm{~N}_{2}{ }^{+} \cdot \mathrm{C}_{7} \mathrm{H}_{5} \mathrm{O}_{2}{ }^{-}$, 2-phenyl- $1 \mathrm{H}$-phenanthro[9,10- $\left.d\right]$ imidazole and benzoic acid form an ion pair complex. The system is consolidated by hydrogen bonds along with $\pi-\pi$ interactions and $\mathrm{N}-\mathrm{H} \cdots \pi$ interactions between the constituent units. For a better understanding of the crystal structure and intermolecular interactions, a Hirshfeld surface analysis was performed.

\section{Chemical context}

When phenanthrene is substituted by a heterocyclic moiety, its intermolecular charge-transfer ability is increased $(\mathrm{Xu}$ et al., 2017). Such a donor- $\pi$-acceptor $(D-\pi-A)$ arrangement has tunable properties that can be controlled by suitable substituents (Cao et al., 2017). The presence of a heteroatom such as $\mathrm{N}$, O or S may give electron-rich heterocycles (thiophene, pyrrole, or furan) or electron-deficient heterocycles (pyridine, phenanthroline) (Xu et al., 2017). The dipole moment and $\lambda_{\max }$ can be modulated by the selection of $D$ and $A$. Thus the photophysical properties can be controlled (Wang et al., 2017). The inclusion of heterocycles enhances the polarizability, thermal and chemical stabilities of such adducts. The $\pi$ conjugated heterocyclic systems increase delocalization, thus enhancing the stability and photophysical properties ( $\mathrm{Gu}$ et al., 2017, Zhang et al., 2012). By proper selection of the heterocyclic substituent, good fluorescence with higher sensitivity can be achieved (Li et al., 2016; Huang et al., 2012). The synthesis of selective chromo-fluorogenic sensors for anions, cations and neutral molecules can be achieved (Chou et al., 2012; Zhuang et al., 2012). Herein we report the crystal structure of the title compound, which was synthesized from 2-phenyl-1 $H$-phenanthro[9,10- $d]$ imidazole and benzoic acid.

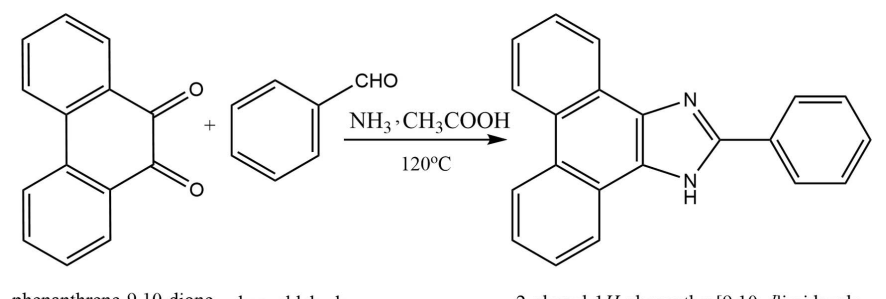




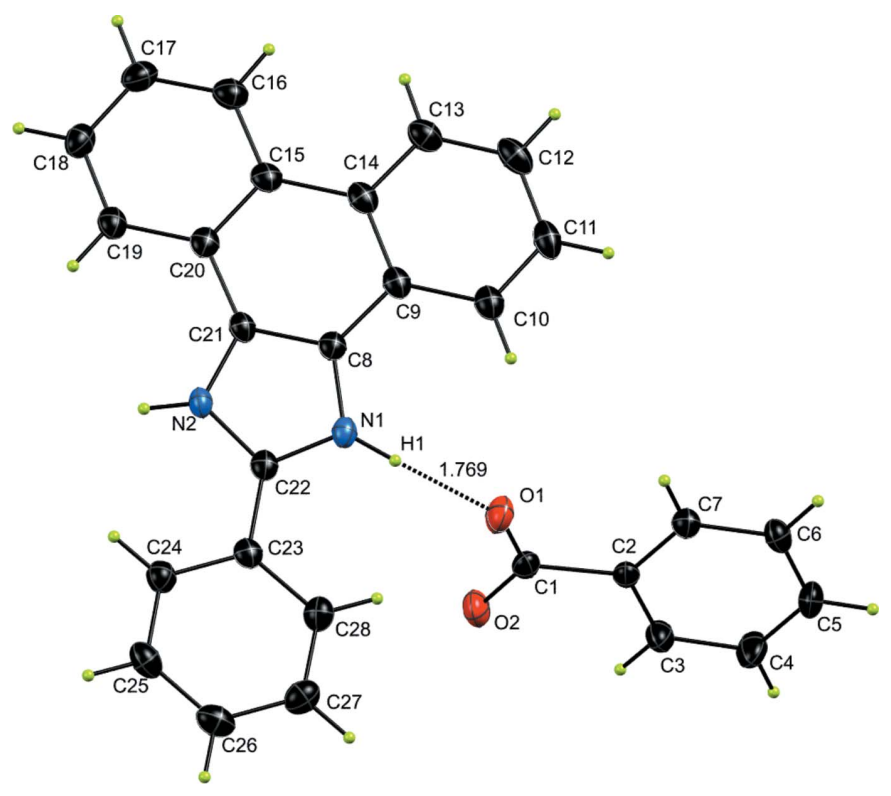

Figure 1

The molecular structure of the title compound with atom labelling. The dashed line indicates the $\mathrm{N}-\mathrm{H} \cdots \mathrm{O}$ hydrogen bond. Displacement ellipsoids are drawn at the $50 \%$ probability level.

\section{Structural commentary}

The structure of the title compound is shown in Fig. 1. The proton from benzoic acid (BA) is completely transferred to the $\mathrm{N}$ atom of the imidazole ring of 2-phenyl-1- $H$-phenanthro[9,10- $d$ ]imidazole (M1), leading to the formation of a $\mathrm{M} 1^{+} \mathrm{BA}^{-}$co-crystal. The space group is monoclinic, $P 2_{1} / n$ and two asymmetric units, two $\mathrm{M}^{+}$ions and two benzoate ions, are combined in an inversion dimer of ion pairs (unit $A$, Fig. 2). The benzoate ion and $\mathrm{M}^{+}$are nearly perpendicular $\left[67.82(4)^{\circ}\right]$ to one another and the torsional angle $\mathrm{C} 1-\mathrm{O} 1-$

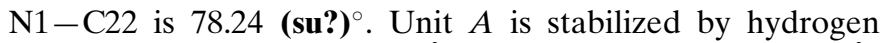
bonds $(\mathrm{N} 1-\mathrm{H} 1 \cdots \mathrm{O} 1,1.77 \AA$, and $\mathrm{N} 2-\mathrm{H} 2 \cdots \mathrm{O} 2,1.83 \AA$; Fig. 2). Beside the hydrogen bonds, there are weak $\pi$ interactions between the two $\mathrm{M}^{+} 1$ moieties [intercentroid separations between the $\mathrm{C} 23-\mathrm{C} 28$ and $\mathrm{C} 8 / \mathrm{C} 9 / \mathrm{C} 14 / \mathrm{C} 15 / \mathrm{C} 20 / \mathrm{C} 21$ rings $=3.4590$ (9) $\AA$ ] .

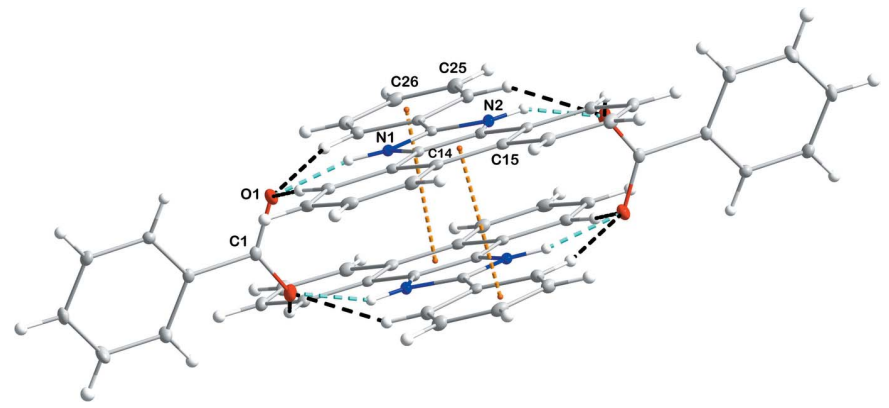

Figure 2

Unit $A$ consisting of two entities each of benzoate ions and M1 moieties, linked by hydrogen bonds and $\pi-\pi$ interactions.
Table 1

Hydrogen-bond geometry $\left(\AA,^{\circ}\right)$.

$\mathrm{Cg} 4$ is the centroid of the $\mathrm{C} 15-\mathrm{C} 20$ benzene ring.

\begin{tabular}{lllll}
\hline$D-\mathrm{H} \cdots A$ & $D-\mathrm{H}$ & $\mathrm{H} \cdots A$ & $D \cdots A$ & $D-\mathrm{H} \cdots A$ \\
\hline $\mathrm{N} 1-\mathrm{H} 1 \cdots \mathrm{O} 1$ & 0.86 & 1.77 & $2.6159(17)$ & 168 \\
$\mathrm{~N} 2-\mathrm{H} 2 \cdots \mathrm{O} 2^{\mathrm{i}}$ & 0.86 & 1.83 & $2.6523(16)$ & 158 \\
$\mathrm{C} 7-\mathrm{H} 7 \cdots C 4^{\mathrm{ii}}$ & 0.93 & 2.79 & $3.585(2)$ & 145 \\
$\mathrm{C} 10-\mathrm{H} 10 \cdots \mathrm{O} 1$ & 0.93 & 2.40 & $3.265(2)$ & 155 \\
$\mathrm{C} 19-\mathrm{H} 19 \cdots \mathrm{O} 2^{\mathrm{i}}$ & 0.93 & 2.54 & $3.372(2)$ & 150 \\
$\mathrm{C} 24-\mathrm{H} 24 \cdots \mathrm{O} 2^{\mathrm{i}}$ & 0.93 & 2.50 & $3.343(2)$ & 152 \\
$\mathrm{C} 28-\mathrm{H} 28 \cdots \mathrm{O} 1$ & 0.93 & 2.48 & $3.365(2)$ & 159 \\
\hline
\end{tabular}

Symmetry codes: (i) $-x+1,-y+1,-z+1$; (ii) $-x,-y+1,-z+1$.

\section{Supramolecular features}

In the crystal, the $A$ units are associated through weak, slipped, $\pi$-stacking interactions between the $\mathrm{C} 9-\mathrm{C} 14$ benzene rings and $\mathrm{N} 1 / \mathrm{C} 22 / \mathrm{N} 2 / \mathrm{C} 21 / \mathrm{C} 8$ imidazole rings across inversion centers [centroid-centroid distance $=3.5675$ (9) $\AA$, dihedral angle $=1.57(8)^{\circ}$, slippage $\left.=1.532 \AA\right)$. The stepped stacks thus formed extend alternately in the directions of the normals to (111) and (1111) and are connected via $\mathrm{C} 7-\mathrm{H} 7 \cdots C g 4$ interactions (Table 1, Fig. 3).

\section{Hirshfeld surface analysis}

The Hirshfeld surfaces provide an extended qualitative and quantitative analysis of the interactions between the constituents of the co-crystal. The analysis shows the presence of $\mathrm{C}-\mathrm{H} \cdots \mathrm{O}$ and $\mathrm{N}-\mathrm{H} \cdots \mathrm{O}$ hydrogen bonds leading to multidirectional interactions to form the three-dimensional structure. The red spots in the Hirshfeld surface (Fig. 4) are centered on the $\mathrm{N} 1-\mathrm{H} 1 \cdots \mathrm{O} 1, \mathrm{C} 10-\mathrm{H} 10 \cdots \mathrm{O} 1$ and $\mathrm{C} 28-$ $\mathrm{H} 28$. O 1 interactions of the benzoate ion with the phenanthrene and with the $\mathrm{N}-\mathrm{H}$ of the imidazole. Their bond lengths are $1.77,2.40$, and $2.48 \AA$, respectively. The fingerprint plots (Fig. 5) show the percentage contribution of the various interactions. Those of $\mathrm{H} \cdots \mathrm{H}$ and $\mathrm{H} \cdots \mathrm{C}$ dominate at $44.8 \%$ and $30.6 \%$, respectively. The $\mathrm{H} \cdots \mathrm{O}$ interactions involve oxygen atoms from the benzoate anion and the $\mathrm{N}-\mathrm{H}$ group of the imidazole ring of $\mathrm{M}^{+}$.

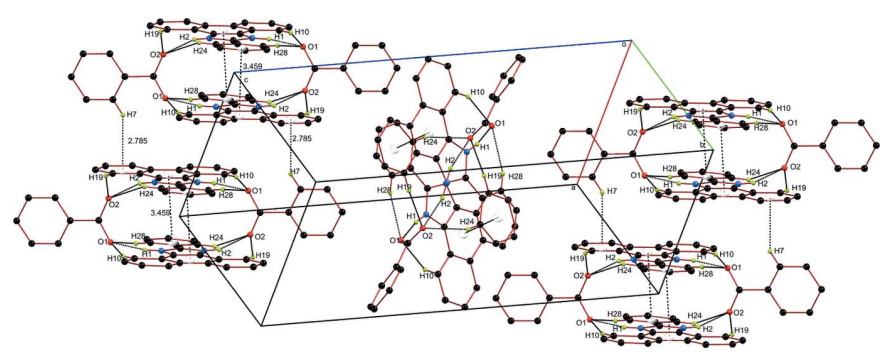

Figure 3

Supramolecular structure showing $A$ units stacked over adjacent rows of $A$ units running perpendicular to each other. 


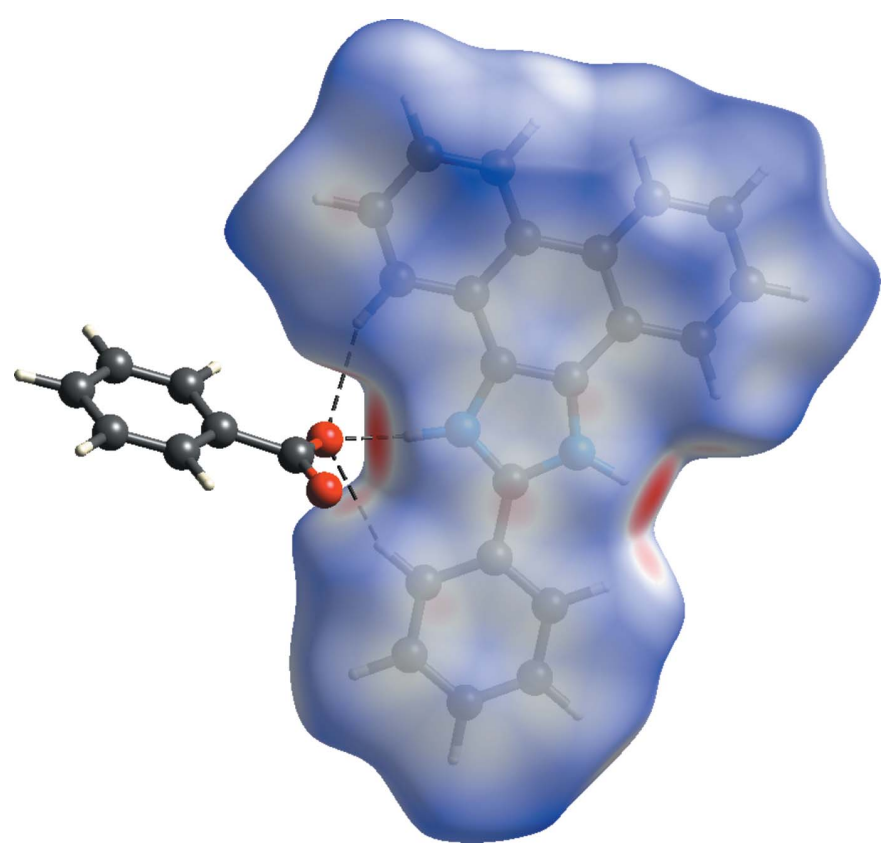

Figure 4

View of the three-dimensional Hirshfeld surface of the title compound plotted over $d_{\text {norm }}$.

\section{Database survey}

A search of the Cambridge Structural database (CSD, version 5.41, update November 2019; Groom et al., 2016) for the 2,3dihydro- $1 H$-phenanthro[9,10- $d$ ] imidazole moiety revealed 45 hits of which the most similar to the title compound are imidazole derivatives (CEZWEL: Mormul et al., 2013; ODEDAD: Li et al., 2016; QORJUD: Tapu et al., 2009; REKXOX: Akula et al., 2017; YUMTEG: Ullah et al., 2009; ZACSAA: Therrien et al., 2014). The $\mathrm{N}-\mathrm{C}$ bond lengths of the imidazole ring in these structures vary from 1.312 (2) to 1.365 (2) $\AA$. The molecular conformations of these structures are also planar.
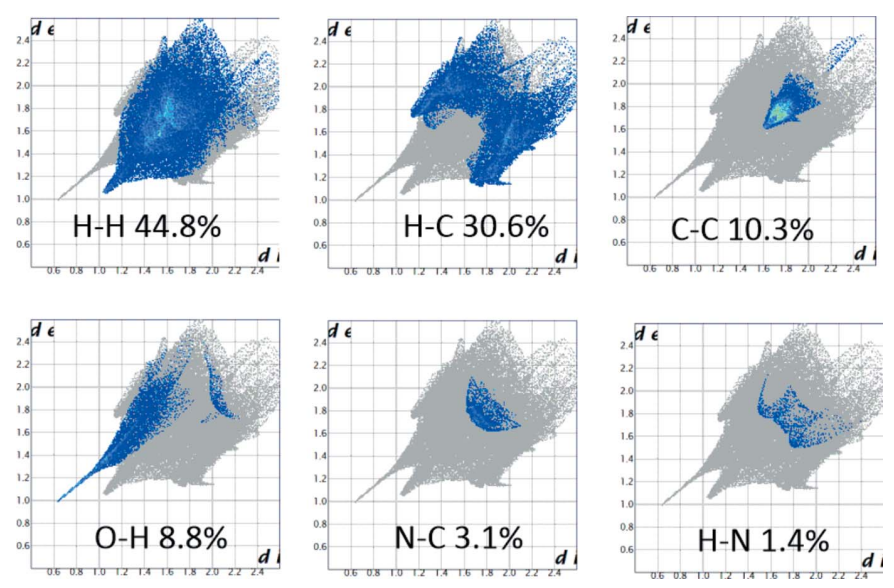

Figure 5

Two-dimensional fingerprint plots of the crystal with the relative contributions of the atom pairs to the Hirshfeld surface.
Table 2

Experimental details.

\begin{tabular}{|c|c|}
\hline \multicolumn{2}{|l|}{ Crystal data } \\
\hline Chemical formula & $\mathrm{C}_{21} \mathrm{H}_{15} \mathrm{~N}_{2}{ }^{+} \cdot \mathrm{C}_{7} \mathrm{H}_{5} \mathrm{O}_{2}^{-}$ \\
\hline$M_{\mathrm{r}}$ & 416.46 \\
\hline Crystal system, space group & Monoclinic, $P 2_{1} / n$ \\
\hline Temperature $(\mathrm{K})$ & 100 \\
\hline$a, b, c(\AA)$ & 9.4693 (4), $8.7384(3), 24.5049$ (9) \\
\hline$\beta\left({ }^{\circ}\right)$ & $91.792(1)$ \\
\hline$V\left(\AA^{6}\right)$ & $2026.70(13)$ \\
\hline$Z$ & 4 \\
\hline Radiation type & Мo $K \alpha$ \\
\hline$\mu\left(\mathrm{mm}^{-1}\right)$ & 0.09 \\
\hline Crystal size $(\mathrm{mm})$ & $0.39 \times 0.28 \times 0.17$ \\
\hline \multicolumn{2}{|l|}{ Data collection } \\
\hline Diffractometer & Bruker APEXII CCD \\
\hline Absorption correction & $\begin{array}{l}\text { Multi-scan (SADABS; Bruker, } \\
\text { 2016) }\end{array}$ \\
\hline$T_{\min }, T_{\max }$ & $0.708,0.746$ \\
\hline $\begin{array}{l}\text { No. of measured, independent and } \\
\text { observed }[I>2 \sigma(I)] \text { reflections }\end{array}$ & $25446,3979,3269$ \\
\hline$R_{\text {int }}$ & 0.046 \\
\hline$(\sin \theta / \lambda)_{\max }\left(\AA^{-1}\right)$ & 0.617 \\
\hline \multicolumn{2}{|l|}{ Refinement } \\
\hline$R\left[F^{2}>2 \sigma\left(F^{2}\right)\right], w R\left(F^{2}\right), S$ & $0.041,0.104,1.10$ \\
\hline No. of reflections & 3979 \\
\hline No. of parameters & 289 \\
\hline $\mathrm{H}$-atom treatment & H-atom parameters constrained \\
\hline$\Delta \rho_{\max }, \Delta \rho_{\min }\left(\mathrm{e} \AA^{-3}\right)$ & $0.23,-0.33$ \\
\hline
\end{tabular}

Computer programs: APEX2 and SAINT (Bruker, 2016), SHELXT2018/3 (Sheldrick, 2015a), SHELXL2018/3 (Sheldrick, 2015b), Mercury (Macrae et al., 2020), WinGX (Farrugia, 2012) and PLATON (Spek, 2020).

\section{Synthesis and crystallization}

A condensation reaction was performed between equimolar quantities of phenanthrene-9,10-dione and benzaldehyde. $1 \mathrm{mmol}$ of phenanthrene-9,10-dione, $1 \mathrm{mmol}$ of benzaldehyde, $5 \mathrm{mmol}$ of ammonium acetate and $30 \mathrm{~mL}$ of glacial acetic acid were added to single-neck $100 \mathrm{~mL}$ round-bottom flask. The mixture was refluxed for $12 \mathrm{~h}$ under nitrogen. After completion of the reaction, the reaction mixture was cooled to room temperature and then $50 \mathrm{~mL}$ of deionized cold water were added. The product precipitated out as pale-brown solid. The solid product was filtered, washed with deionized water and dried in a vacuum oven to give 2-phenyl- $1 H$-phenanthro[9,10d] imidazole (M1) as the final product. Crystals were prepared using $20 \mathrm{mg}$ of M1 and $20 \mathrm{mg}$ of benzoic acid dissolved in $5 \mathrm{~mL}$ of ethanol. The clear solution was left undisturbed for crystallization. Fine crystals were obtained after 15 days.

\section{Refinement}

Crystal data, data collection and structure refinement details are summarized in Table 2. The NH hydrogen atoms were located in difference-Fourier maps and, together with the carbon-bound hydrogen atoms, were included as riding contributions in calculated positions $[\mathrm{N}-\mathrm{H}=0.86, \mathrm{C}-\mathrm{H}=$ $\left.0.93 \AA ; U_{\text {iso }}(\mathrm{H})=1.2 U_{\text {eq }}(\mathrm{C}, \mathrm{N})\right]$. 


\section{Acknowledgements}

The authors are grateful to the Department of Applied Chemistry, Aligarh Muslim University, Aligarh, India, for providing laboratory facilities.

\section{Funding information}

Dr Farman Ali acknowledges the DST for INSPIRE Faculty Funding.

\section{References}

Akula, S. B., Chen, H.-S., Su, C., Chen, B.-R., Chiou, J.-J., Shieh, C.-H., Lin, Y.-F. \& Li, W.-R. (2017). Inorg. Chem. 56, 12987-12995.

Bruker (2016). APEX2, SAINT and SADABS. Bruker AXS Inc., Madison, Wisconsin, USA.

Cao, L., Zhang, D., Xu, L., Fang, Z., Jiang, X.-F. \& Lu, F. (2017). Eur. J. Org. Chem. 2017, 2495-2500.

Chou, H. H., Chen, Y. H., Hsu, H. P., Chang, W. H., Chen, Y. H. \& Cheng, C. H. (2012). Adv. Mater. 24, 5867-5871.

Farrugia, L. J. (2012). J. Appl. Cryst. 45, 849-854.

Groom, C. R., Bruno, I. J., Lightfoot, M. P. \& Ward, S. C. (2016). Acta Cryst. B72, 171-179.

Gu, P.-Y., Wang, Z., Liu, G., Yao, H., Wang, Z., Li, Y., Zhu, J., Li, S. \& Zhang, Q. (2017). Chem. Mater. 29, 4172-4175.
Huang, H., Wang, Y., Zhuang, S., Yang, X., Wang, L. \& Yang, C. (2012). J. Phys. Chem. C, 116, 19458-19466.

Li, J., Chen, S., Wang, Z. \& Zhang, Q. (2016). Chem. Rec. 16, 15181530.

Macrae, C. F., Sovago, I., Cottrell, S. J., Galek, P. T. A., McCabe, P., Pidcock, E., Platings, M., Shields, G. P., Stevens, J. S., Towler, M. \& Wood, P. A. (2020). J. Appl. Cryst. 53, 226-235.

Mormul, J., Steimann, M., Maichle-Mössmer, C. \& Nagel, U. (2013). Eur. J. Inorg. Chem. pp. 3421-3428.

Sheldrick, G. M. (2015a). Acta Cryst. A71, 3-8.

Sheldrick, G. M. (2015b). Acta Cryst. C71, 3-8.

Spek, A. L. (2020). Acta Cryst. E76, 1-11.

Tapu, D., Owens, C., VanDerveer, D. \& Gwaltney, K. (2009). Organometallics, 28, 270-276.

Therrien, J. A., Wolf, M. O. \& Patrick, B. O. (2014). Inorg. Chem. 53, 12962-12972.

Ullah, F., Kindermann, M. K., Jones, P. G. \& Heinicke, J. (2009). Organometallics, 28, 2441-2449.

Wang, Z., Gu, P., Liu, G., Yao, H., Wu, Y., Li, Y., Rakesh, G., Zhu, J., Fu, H. \& Zhang, Q. (2017). Chem. Commun. 53, 7772-7775.

Xu, L., Zhang, D., Zhou, Y., Zheng, Y., Cao, L., Jiang, X.-F. \& Lu, F. (2017). Opt. Mater. 70, 131-137.

Zhang, Y., Lai, S.-L., Tong, Q.-X., Lo, M.-F., Ng, T.-W., Chan, M.-Y., Wen, Z.-C., He, J., Jeff, K.-S., Tang, X.-L., Liu, W., Ko, C., Wang, P. \& Lee, C. (2012). Chem. Mater. 24, 61-70.

Zhuang, S., Shangguan, R., Jin, J., Tu, G., Wang, L., Chen, J., Ma, D. \& Zhu, X. (2012). Org. Electron. 13, 3050-3059. 


\section{supporting information}

Acta Cryst. (2020). E76, 724-727 [https://doi.org/10.1107/S2056989020005344]

Crystal structure and Hirshfeld surface analysis of 2-phenyl-1Hphenanthro[9,10- $d]$ imidazol-3-ium benzoate

\section{Ruby Ahmed, Onur Erman Doğan, Farman Ali, Musheer Ahmad, Adeeba Ahmed, Necmi Dege} and Irina A. Golenia

Computing details

Data collection: APEX2 (Bruker, 2016); cell refinement: SAINT (Bruker, 2016); data reduction: SAINT (Bruker, 2016); program(s) used to solve structure: SHELXT2018/3 (Sheldrick, 2015a); program(s) used to refine structure:

SHELXL2018/3 (Sheldrick, 2015b); molecular graphics: Mercury (Macrae et al., 2020); software used to prepare material for publication: WinGX (Farrugia, 2012) and PLATON (Spek, 2020).

2-Phenyl-1H-phenanthro[9,10-d]imidazol-3-ium benzoate

Crystal data

$\mathrm{C}_{21} \mathrm{H}_{15} \mathrm{~N}_{2}^{+} \cdot \mathrm{C}_{7} \mathrm{H}_{5} \mathrm{O}_{2}^{-}$

$M_{r}=416.46$

Monoclinic, $P 2_{1} / n$

$a=9.4693(4) \AA$

$b=8.7384(3) \AA$

$c=24.5049$ (9) $\AA$

$\beta=91.792(1)^{\circ}$

$V=2026.70(13) \AA^{3}$

$Z=4$

\section{Data collection}

Bruker APEXII CCD

diffractometer

$\varphi$ and $\omega$ scans

Absorption correction: multi-scan

(SADABS; Bruker, 2016)

$T_{\text {min }}=0.708, T_{\text {max }}=0.746$

25446 measured reflections

Refinement

Refinement on $F^{2}$

Least-squares matrix: full

$R\left[F^{2}>2 \sigma\left(F^{2}\right)\right]=0.041$

$w R\left(F^{2}\right)=0.104$

$S=1.10$

3979 reflections

289 parameters

0 restraints

Primary atom site location: iterative
$F(000)=872$

$D_{\mathrm{x}}=1.365 \mathrm{Mg} \mathrm{m}^{-3}$

Mo $K \alpha$ radiation, $\lambda=0.71073 \AA$

Cell parameters from 9121 reflections

$\theta=3.2-28.1^{\circ}$

$\mu=0.09 \mathrm{~mm}^{-1}$

$T=100 \mathrm{~K}$

Block, pink

$0.39 \times 0.28 \times 0.17 \mathrm{~mm}$

3979 independent reflections

3269 reflections with $I>2 \sigma(I)$

$R_{\text {int }}=0.046$

$\theta_{\max }=26.0^{\circ}, \theta_{\min }=2.3^{\circ}$

$h=-11 \rightarrow 11$

$k=-10 \rightarrow 10$

$l=-30 \rightarrow 30$

Hydrogen site location: inferred from neighbouring sites

$\mathrm{H}$-atom parameters constrained

$w=1 /\left[\sigma^{2}\left(F_{\mathrm{o}}^{2}\right)+(0.0356 P)^{2}+1.042 P\right]$

where $P=\left(F_{\mathrm{o}}^{2}+2 F_{\mathrm{c}}^{2}\right) / 3$

$(\Delta / \sigma)_{\max }<0.001$

$\Delta \rho_{\max }=0.23$ e $\AA^{-3}$

$\Delta \rho_{\min }=-0.33$ e $\AA^{-3}$ 


\section{Special details}

Geometry. All esds (except the esd in the dihedral angle between two 1.s. planes) are estimated using the full covariance matrix. The cell esds are taken into account individually in the estimation of esds in distances, angles and torsion angles; correlations between esds in cell parameters are only used when they are defined by crystal symmetry. An approximate (isotropic) treatment of cell esds is used for estimating esds involving l.s. planes.

Fractional atomic coordinates and isotropic or equivalent isotropic displacement parameters $\left(\hat{A}^{2}\right)$

\begin{tabular}{|c|c|c|c|c|}
\hline & $x$ & $y$ & $z$ & $U_{\text {iso }} * / U_{\text {eq }}$ \\
\hline $\mathrm{O} 1$ & $0.23573(12)$ & $0.35667(14)$ & $0.39271(5)$ & $0.0272(3)$ \\
\hline $\mathrm{O} 2$ & $0.43116(12)$ & $0.21182(14)$ & $0.39047(5)$ & $0.0277(3)$ \\
\hline N1 & $0.27589(13)$ & $0.51384(15)$ & $0.48272(5)$ & $0.0174(3)$ \\
\hline H1 & 0.259534 & 0.474240 & 0.451017 & $0.021^{*}$ \\
\hline N2 & $0.36868(13)$ & $0.65362(15)$ & $0.54849(5)$ & $0.0177(3)$ \\
\hline $\mathrm{H} 2$ & 0.421794 & 0.718335 & 0.565817 & $0.021^{*}$ \\
\hline $\mathrm{C} 1$ & $0.30537(16)$ & $0.24344(19)$ & $0.37667(6)$ & $0.0190(3)$ \\
\hline $\mathrm{C} 2$ & $0.23159(16)$ & $0.13699(18)$ & $0.33666(6)$ & $0.0174(3)$ \\
\hline $\mathrm{C} 3$ & 0.30647 (17) & $0.0201(2)$ & $0.31269(7)$ & $0.0260(4)$ \\
\hline H3 & 0.400888 & 0.004987 & 0.322848 & $0.031^{*}$ \\
\hline $\mathrm{C} 4$ & $0.24293(19)$ & $-0.0746(2)$ & $0.27379(7)$ & $0.0327(4)$ \\
\hline $\mathrm{H} 4$ & 0.294737 & -0.151762 & 0.257587 & $0.039^{*}$ \\
\hline $\mathrm{C} 5$ & $0.10194(18)$ & $-0.0537(2)$ & $0.25920(7)$ & $0.0261(4)$ \\
\hline H5 & 0.059013 & -0.115598 & 0.232633 & $0.031^{*}$ \\
\hline C6 & $0.02506(17)$ & $0.05930(19)$ & $0.28422(7)$ & $0.0225(4)$ \\
\hline H6 & -0.070461 & 0.071109 & 0.275216 & $0.027^{*}$ \\
\hline C7 & $0.08921(16)$ & $0.15524(18)$ & $0.32262(6)$ & $0.0194(3)$ \\
\hline H7 & 0.037017 & 0.231785 & 0.338992 & $0.023^{*}$ \\
\hline $\mathrm{C} 8$ & $0.20749(15)$ & $0.47649(18)$ & $0.52979(6)$ & $0.0172(3)$ \\
\hline C9 & $0.09665(15)$ & $0.36873(18)$ & $0.53830(6)$ & $0.0185(3)$ \\
\hline $\mathrm{C} 10$ & $0.03891(16)$ & $0.27684(18)$ & $0.49600(7)$ & $0.0214(4)$ \\
\hline H10 & 0.070829 & 0.286745 & 0.460668 & $0.026^{*}$ \\
\hline $\mathrm{C} 11$ & $-0.06499(16)$ & $0.17229(19)$ & $0.50718(7)$ & $0.0241(4)$ \\
\hline H11 & -0.102982 & 0.111124 & 0.479346 & $0.029^{*}$ \\
\hline $\mathrm{C} 12$ & $-0.11351(17)$ & 0.1577 (2) & $0.56009(7)$ & $0.0267(4)$ \\
\hline H12 & -0.183127 & 0.086184 & 0.567421 & $0.032^{*}$ \\
\hline $\mathrm{C} 13$ & $-0.05897(17)$ & $0.2486(2)$ & $0.60154(7)$ & $0.0243(4)$ \\
\hline H13 & -0.093418 & 0.238283 & 0.636479 & $0.029^{*}$ \\
\hline C14 & $0.04783(15)$ & $0.35686(18)$ & $0.59221(7)$ & $0.0201(3)$ \\
\hline $\mathrm{C} 15$ & $0.10846(15)$ & $0.45281(18)$ & $0.63613(6)$ & $0.0197(3)$ \\
\hline $\mathrm{C} 16$ & $0.06231(17)$ & $0.4443(2)$ & $0.69008(7)$ & $0.0244(4)$ \\
\hline H16 & -0.010480 & 0.377451 & 0.698225 & $0.029^{*}$ \\
\hline C17 & $0.12253(17)$ & $0.5328(2)$ & $0.73112(7)$ & $0.0263(4)$ \\
\hline H17 & 0.090928 & 0.523517 & 0.766521 & $0.032^{*}$ \\
\hline $\mathrm{C} 18$ & $0.23025(17)$ & $0.6361(2)$ & $0.72023(7)$ & $0.0243(4)$ \\
\hline H18 & 0.269913 & 0.695635 & 0.748191 & $0.029^{*}$ \\
\hline C19 & $0.27759(16)$ & $0.64963(19)$ & $0.66800(6)$ & $0.0213(4)$ \\
\hline H19 & 0.348675 & 0.719290 & 0.660521 & $0.026^{*}$ \\
\hline $\mathrm{C} 20$ & $0.21874(15)$ & $0.55833(18)$ & $0.62580(6)$ & $0.0187(3)$ \\
\hline
\end{tabular}




\begin{tabular}{lllll} 
C21 & $0.26552(15)$ & $0.56429(18)$ & $0.57097(6)$ & $0.0173(3)$ \\
C22 & $0.37202(15)$ & $0.62210(18)$ & $0.49467(6)$ & $0.0174(3)$ \\
C23 & $0.46252(15)$ & $0.69837(18)$ & $0.45586(6)$ & $0.0180(3)$ \\
C24 & $0.55209(16)$ & $0.81660(19)$ & $0.47343(7)$ & $0.0224(4)$ \\
H24 & 0.554159 & 0.846890 & 0.509828 & $0.027^{*}$ \\
C25 & $0.63769(17)$ & $0.8886(2)$ & $0.43664(7)$ & $0.0264(4)$ \\
H25 & 0.697088 & 0.967356 & 0.448523 & $0.032^{*}$ \\
C26 & $0.63607(17)$ & $0.8448(2)$ & $0.38243(7)$ & $0.0264(4)$ \\
H26 & 0.695022 & 0.892778 & 0.358048 & $0.032^{*}$ \\
C27 & $0.54566(18)$ & $0.7287(2)$ & $0.36468(7)$ & $0.0260(4)$ \\
H27 & 0.543584 & 0.699591 & 0.328159 & $0.031^{*}$ \\
C28 & $0.45880(17)$ & $0.65616(19)$ & $0.40079(7)$ & $0.0224(4)$ \\
H28 & 0.397852 & 0.579232 & 0.388489 & $0.027^{*}$ \\
\hline
\end{tabular}

Atomic displacement parameters $\left(\AA^{2}\right)$

\begin{tabular}{|c|c|c|c|c|c|c|}
\hline & $U^{11}$ & $U^{22}$ & $U^{33}$ & $U^{12}$ & $U^{13}$ & $U^{23}$ \\
\hline $\mathrm{O} 1$ & $0.0306(6)$ & $0.0247(6)$ & $0.0259(6)$ & $-0.0004(5)$ & $-0.0033(5)$ & -0.0090 \\
\hline $\mathrm{O} 2$ & $0.0214(6)$ & $0.0332(7)$ & $0.0279(6)$ & $-0.0035(5)$ & $-0.0069(5)$ & $-0.0051(5)$ \\
\hline N1 & $0.0161(6)$ & $0.0172(7)$ & $0.0185(7)$ & $0.0026(5)$ & $-0.0037(5)$ & -0.0023 \\
\hline N2 & $0.0151(6)$ & $0.0177(7)$ & $0.0200(7)$ & $-0.0006(5)$ & $-0.0035(5)$ & $-0.0032(5)$ \\
\hline $\mathrm{C} 1$ & $0.0211(8)$ & $0.0216(8)$ & $0.0144(7)$ & $-0.0032(7)$ & $0.0002(6)$ & $0.0011(6)$ \\
\hline $\mathrm{C} 2$ & $0.0184(7)$ & $0.0192(8)$ & $0.0146(7)$ & $-0.0039(6)$ & $0.0010(6)$ & $0.0011(6)$ \\
\hline $\mathrm{C} 3$ & $0.0166(7)$ & $0.0336(10)$ & 0.0277 (9) & $0.0000(7)$ & $-0.0019(7)$ & $-0.0089(8)$ \\
\hline $\mathrm{C} 4$ & $0.0264(9)$ & $0.0371(11)$ & $0.0343(10)$ & $0.0034(8)$ & $-0.0006(8)$ & $-0.0185(9)$ \\
\hline $\mathrm{C} 5$ & $0.0269(9)$ & $0.0285(9)$ & $0.0226(8)$ & $-0.0054(7)$ & $-0.0046(7)$ & $-0.0076(7)$ \\
\hline C6 & $0.0180(7)$ & $0.0245(9)$ & $0.0245(8)$ & $-0.0022(7)$ & $-0.0051(7)$ & $0.0006(7)$ \\
\hline $\mathrm{C} 7$ & $0.0192(8)$ & $0.0187(8)$ & $0.0203(8)$ & $0.0010(6)$ & $-0.0007(6)$ & $-0.0003(6)$ \\
\hline $\mathrm{C} 8$ & $0.0145(7)$ & $0.0173(8)$ & $0.0197(8)$ & $0.0042(6)$ & $-0.0019(6)$ & $-0.0001(6)$ \\
\hline C9 & $0.0143(7)$ & $0.0156(8)$ & $0.0254(8)$ & $0.0045(6)$ & $-0.0036(6)$ & $0.0004(6)$ \\
\hline $\mathrm{C} 10$ & $0.0177(8)$ & $0.0183(8)$ & $0.0281(9)$ & $0.0042(6)$ & $-0.0035(7)$ & $-0.0017(7)$ \\
\hline $\mathrm{C} 11$ & $0.0182(8)$ & $0.0171(8)$ & $0.0364(10)$ & $0.0028(7)$ & $-0.0075(7)$ & $-0.0035(7)$ \\
\hline $\mathrm{C} 12$ & $0.0178(8)$ & $0.0200(9)$ & $0.0420(11)$ & $0.0001(7)$ & $-0.0019(7)$ & $0.0058(8)$ \\
\hline C13 & $0.0185(8)$ & $0.0255(9)$ & $0.0288(9)$ & $0.0015(7)$ & $-0.0009(7)$ & $0.0054(7)$ \\
\hline $\mathrm{C} 14$ & $0.0141(7)$ & $0.0181(8)$ & $0.0278(9)$ & $0.0046(6)$ & $-0.0025(6)$ & $0.0019(7)$ \\
\hline $\mathrm{C} 15$ & $0.0147(7)$ & $0.0207(8)$ & $0.0234(8)$ & $0.0060(6)$ & $-0.0007(6)$ & $0.0013(7)$ \\
\hline $\mathrm{C} 16$ & $0.0192(8)$ & $0.0275(9)$ & $0.0264(9)$ & $0.0037(7)$ & $0.0013(7)$ & $0.0027(7)$ \\
\hline $\mathrm{C} 17$ & $0.0241(8)$ & $0.0347(10)$ & $0.0201(8)$ & $0.0076(8)$ & $0.0022(7)$ & $0.0002(7)$ \\
\hline C18 & $0.0217(8)$ & $0.0299(9)$ & $0.0212(8)$ & $0.0068(7)$ & $-0.0044(7)$ & $-0.0032(7)$ \\
\hline C19 & $0.0162(7)$ & $0.0227(9)$ & $0.0247(9)$ & $0.0040(6)$ & $-0.0035(6)$ & $-0.0023(7)$ \\
\hline $\mathrm{C} 20$ & $0.0152(7)$ & $0.0190(8)$ & $0.0217(8)$ & $0.0064(6)$ & $-0.0033(6)$ & $-0.0002(6)$ \\
\hline $\mathrm{C} 21$ & $0.0137(7)$ & $0.0166(8)$ & $0.0214(8)$ & $0.0026(6)$ & $-0.0032(6)$ & $0.0000(6)$ \\
\hline $\mathrm{C} 22$ & $0.0150(7)$ & $0.0157(8)$ & $0.0214(8)$ & $0.0051(6)$ & $-0.0032(6)$ & $-0.0016(6)$ \\
\hline $\mathrm{C} 23$ & $0.0144(7)$ & $0.0171(8)$ & $0.0224(8)$ & $0.0051(6)$ & $-0.0021(6)$ & $0.0009(6)$ \\
\hline $\mathrm{C} 24$ & $0.0190(8)$ & $0.0243(9)$ & $0.0236(8)$ & $0.0014(7)$ & $-0.0024(7)$ & $-0.0011(7)$ \\
\hline $\mathrm{C} 25$ & $0.0194(8)$ & $0.0265(9)$ & $0.0330(10)$ & $-0.0021(7)$ & $-0.0045(7)$ & $0.0038(8)$ \\
\hline $\mathrm{C} 26$ & $0.0203(8)$ & $0.0286(10)$ & $0.0304(9)$ & $0.0034(7)$ & $0.0014(7)$ & $0.0097(8)$ \\
\hline $\mathrm{C} 27$ & $0.0301(9)$ & $0.0264(9)$ & $0.0215(8)$ & $0.0057(7)$ & $0.0000(7)$ & $0.0018(7)$ \\
\hline
\end{tabular}




$\begin{array}{lllllll}\mathrm{C} 28 & 0.0234(8) & 0.0191(8) & 0.0243(8) & 0.0021(7) & -0.0029(7) & -0.0011(7)\end{array}$

Geometric parameters $(\AA, \stackrel{o}{)}$

\begin{tabular}{|c|c|c|c|}
\hline $\mathrm{O} 1-\mathrm{C} 1$ & $1.2588(19)$ & $\mathrm{C} 12-\mathrm{C} 13$ & $1.377(2)$ \\
\hline $\mathrm{O} 2-\mathrm{C} 1$ & $1.2587(19)$ & $\mathrm{C} 12-\mathrm{H} 12$ & 0.9300 \\
\hline $\mathrm{N} 1-\mathrm{C} 22$ & $1.339(2)$ & $\mathrm{C} 13-\mathrm{C} 14$ & $1.409(2)$ \\
\hline $\mathrm{N} 1-\mathrm{C} 8$ & $1.380(2)$ & $\mathrm{C} 13-\mathrm{H} 13$ & 0.9300 \\
\hline $\mathrm{N} 1-\mathrm{H} 1$ & 0.8600 & $\mathrm{C} 14-\mathrm{C} 15$ & $1.467(2)$ \\
\hline $\mathrm{N} 2-\mathrm{C} 22$ & $1.349(2)$ & $\mathrm{C} 15-\mathrm{C} 16$ & 1.407 (2) \\
\hline $\mathrm{N} 2-\mathrm{C} 21$ & $1.379(2)$ & $\mathrm{C} 15-\mathrm{C} 20$ & $1.422(2)$ \\
\hline $\mathrm{N} 2-\mathrm{H} 2$ & 0.8600 & $\mathrm{C} 16-\mathrm{C} 17$ & $1.378(2)$ \\
\hline $\mathrm{C} 1-\mathrm{C} 2$ & 1.507 (2) & $\mathrm{C} 16-\mathrm{H} 16$ & 0.9300 \\
\hline $\mathrm{C} 2-\mathrm{C} 3$ & $1.384(2)$ & $\mathrm{C} 17-\mathrm{C} 18$ & $1.394(2)$ \\
\hline $\mathrm{C} 2-\mathrm{C} 7$ & $1.390(2)$ & C17-H17 & 0.9300 \\
\hline $\mathrm{C} 3-\mathrm{C} 4$ & $1.386(2)$ & $\mathrm{C} 18-\mathrm{C} 19$ & $1.374(2)$ \\
\hline $\mathrm{C} 3-\mathrm{H} 3$ & 0.9300 & $\mathrm{C} 18-\mathrm{H} 18$ & 0.9300 \\
\hline $\mathrm{C} 4-\mathrm{C} 5$ & $1.383(2)$ & $\mathrm{C} 19-\mathrm{C} 20$ & $1.407(2)$ \\
\hline $\mathrm{C} 4-\mathrm{H} 4$ & 0.9300 & C19-H19 & 0.9300 \\
\hline $\mathrm{C} 5-\mathrm{C} 6$ & $1.382(2)$ & $\mathrm{C} 20-\mathrm{C} 21$ & $1.429(2)$ \\
\hline $\mathrm{C} 5-\mathrm{H} 5$ & 0.9300 & $\mathrm{C} 22-\mathrm{C} 23$ & $1.461(2)$ \\
\hline $\mathrm{C} 6-\mathrm{C} 7$ & $1.386(2)$ & $\mathrm{C} 23-\mathrm{C} 24$ & $1.396(2)$ \\
\hline $\mathrm{C} 6-\mathrm{H} 6$ & 0.9300 & $\mathrm{C} 23-\mathrm{C} 28$ & $1.398(2)$ \\
\hline $\mathrm{C} 7-\mathrm{H} 7$ & 0.9300 & $\mathrm{C} 24-\mathrm{C} 25$ & $1.382(2)$ \\
\hline $\mathrm{C} 8-\mathrm{C} 21$ & $1.369(2)$ & $\mathrm{C} 24-\mathrm{H} 24$ & 0.9300 \\
\hline $\mathrm{C} 8-\mathrm{C} 9$ & $1.430(2)$ & $\mathrm{C} 25-\mathrm{C} 26$ & $1.382(2)$ \\
\hline $\mathrm{C} 9-\mathrm{C} 10$ & $1.408(2)$ & $\mathrm{C} 25-\mathrm{H} 25$ & 0.9300 \\
\hline $\mathrm{C} 9-\mathrm{C} 14$ & $1.417(2)$ & $\mathrm{C} 26-\mathrm{C} 27$ & $1.389(2)$ \\
\hline $\mathrm{C} 10-\mathrm{C} 11$ & $1.376(2)$ & $\mathrm{C} 26-\mathrm{H} 26$ & 0.9300 \\
\hline $\mathrm{C} 10-\mathrm{H} 10$ & 0.9300 & $\mathrm{C} 27-\mathrm{C} 28$ & $1.381(2)$ \\
\hline $\mathrm{C} 11-\mathrm{C} 12$ & $1.395(2)$ & $\mathrm{C} 27-\mathrm{H} 27$ & 0.9300 \\
\hline $\mathrm{C} 11-\mathrm{H} 11$ & 0.9300 & $\mathrm{C} 28-\mathrm{H} 28$ & 0.9300 \\
\hline $\mathrm{C} 22-\mathrm{N} 1-\mathrm{C} 8$ & $108.53(13)$ & $\mathrm{C} 13-\mathrm{C} 14-\mathrm{C} 9$ & $117.21(15)$ \\
\hline $\mathrm{C} 22-\mathrm{N} 1-\mathrm{H} 1$ & 125.7 & $\mathrm{C} 13-\mathrm{C} 14-\mathrm{C} 15$ & $122.08(15)$ \\
\hline $\mathrm{C} 8-\mathrm{N} 1-\mathrm{H} 1$ & 125.7 & $\mathrm{C} 9-\mathrm{C} 14-\mathrm{C} 15$ & $120.70(14)$ \\
\hline $\mathrm{C} 22-\mathrm{N} 2-\mathrm{C} 21$ & $108.28(13)$ & $\mathrm{C} 16-\mathrm{C} 15-\mathrm{C} 20$ & $116.92(15)$ \\
\hline $\mathrm{C} 22-\mathrm{N} 2-\mathrm{H} 2$ & 125.9 & $\mathrm{C} 16-\mathrm{C} 15-\mathrm{C} 14$ & $122.22(15)$ \\
\hline $\mathrm{C} 21-\mathrm{N} 2-\mathrm{H} 2$ & 125.9 & $\mathrm{C} 20-\mathrm{C} 15-\mathrm{C} 14$ & $120.86(14)$ \\
\hline $\mathrm{O} 2-\mathrm{C} 1-\mathrm{O} 1$ & $126.11(15)$ & $\mathrm{C} 17-\mathrm{C} 16-\mathrm{C} 15$ & $121.50(16)$ \\
\hline $\mathrm{O} 2-\mathrm{C} 1-\mathrm{C} 2$ & $117.05(14)$ & $\mathrm{C} 17-\mathrm{C} 16-\mathrm{H} 16$ & 119.3 \\
\hline $\mathrm{O} 1-\mathrm{C} 1-\mathrm{C} 2$ & $116.84(14)$ & $\mathrm{C} 15-\mathrm{C} 16-\mathrm{H} 16$ & 119.3 \\
\hline $\mathrm{C} 3-\mathrm{C} 2-\mathrm{C} 7$ & $119.04(14)$ & $\mathrm{C} 16-\mathrm{C} 17-\mathrm{C} 18$ & $120.82(16)$ \\
\hline $\mathrm{C} 3-\mathrm{C} 2-\mathrm{C} 1$ & $119.87(14)$ & $\mathrm{C} 16-\mathrm{C} 17-\mathrm{H} 17$ & 119.6 \\
\hline $\mathrm{C} 7-\mathrm{C} 2-\mathrm{C} 1$ & $121.09(14)$ & $\mathrm{C} 18-\mathrm{C} 17-\mathrm{H} 17$ & 119.6 \\
\hline $\mathrm{C} 2-\mathrm{C} 3-\mathrm{C} 4$ & $121.02(15)$ & $\mathrm{C} 19-\mathrm{C} 18-\mathrm{C} 17$ & $119.72(16)$ \\
\hline $\mathrm{C} 2-\mathrm{C} 3-\mathrm{H} 3$ & 119.5 & $\mathrm{C} 19-\mathrm{C} 18-\mathrm{H} 18$ & 120.1 \\
\hline $\mathrm{C} 4-\mathrm{C} 3-\mathrm{H} 3$ & 119.5 & $\mathrm{C} 17-\mathrm{C} 18-\mathrm{H} 18$ & 120.1 \\
\hline
\end{tabular}




\begin{tabular}{|c|c|c|c|}
\hline $\mathrm{C} 5-\mathrm{C} 4-\mathrm{C} 3$ & $119.58(16)$ & $\mathrm{C} 18-\mathrm{C} 19-\mathrm{C} 20$ & $120.15(16)$ \\
\hline $\mathrm{C} 5-\mathrm{C} 4-\mathrm{H} 4$ & 120.2 & $\mathrm{C} 18-\mathrm{C} 19-\mathrm{H} 19$ & 119.9 \\
\hline $\mathrm{C} 3-\mathrm{C} 4-\mathrm{H} 4$ & 120.2 & $\mathrm{C} 20-\mathrm{C} 19-\mathrm{H} 19$ & 119.9 \\
\hline $\mathrm{C} 6-\mathrm{C} 5-\mathrm{C} 4$ & $119.83(15)$ & $\mathrm{C} 19-\mathrm{C} 20-\mathrm{C} 15$ & $120.88(15)$ \\
\hline $\mathrm{C} 6-\mathrm{C} 5-\mathrm{H} 5$ & 120.1 & $\mathrm{C} 19-\mathrm{C} 20-\mathrm{C} 21$ & $122.89(15)$ \\
\hline $\mathrm{C} 4-\mathrm{C} 5-\mathrm{H} 5$ & 120.1 & $\mathrm{C} 15-\mathrm{C} 20-\mathrm{C} 21$ & $116.23(14)$ \\
\hline $\mathrm{C} 5-\mathrm{C} 6-\mathrm{C} 7$ & $120.51(15)$ & $\mathrm{C} 8-\mathrm{C} 21-\mathrm{N} 2$ & $107.21(14)$ \\
\hline $\mathrm{C} 5-\mathrm{C} 6-\mathrm{H} 6$ & 119.7 & $\mathrm{C} 8-\mathrm{C} 21-\mathrm{C} 20$ & $122.93(14)$ \\
\hline $\mathrm{C} 7-\mathrm{C} 6-\mathrm{H} 6$ & 119.7 & $\mathrm{~N} 2-\mathrm{C} 21-\mathrm{C} 20$ & $129.85(14)$ \\
\hline $\mathrm{C} 6-\mathrm{C} 7-\mathrm{C} 2$ & $119.97(15)$ & $\mathrm{N} 1-\mathrm{C} 22-\mathrm{N} 2$ & $108.77(14)$ \\
\hline $\mathrm{C} 6-\mathrm{C} 7-\mathrm{H} 7$ & 120.0 & $\mathrm{~N} 1-\mathrm{C} 22-\mathrm{C} 23$ & $126.05(14)$ \\
\hline $\mathrm{C} 2-\mathrm{C} 7-\mathrm{H} 7$ & 120.0 & $\mathrm{~N} 2-\mathrm{C} 22-\mathrm{C} 23$ & $125.15(14)$ \\
\hline $\mathrm{C} 21-\mathrm{C} 8-\mathrm{N} 1$ & $107.20(13)$ & $\mathrm{C} 24-\mathrm{C} 23-\mathrm{C} 28$ & $119.31(15)$ \\
\hline $\mathrm{C} 21-\mathrm{C} 8-\mathrm{C} 9$ & $122.75(15)$ & $\mathrm{C} 24-\mathrm{C} 23-\mathrm{C} 22$ & $119.99(14)$ \\
\hline $\mathrm{N} 1-\mathrm{C} 8-\mathrm{C} 9$ & $130.06(14)$ & $\mathrm{C} 28-\mathrm{C} 23-\mathrm{C} 22$ & $120.69(14)$ \\
\hline $\mathrm{C} 10-\mathrm{C} 9-\mathrm{C} 14$ & $120.94(15)$ & $\mathrm{C} 25-\mathrm{C} 24-\mathrm{C} 23$ & $119.90(16)$ \\
\hline $\mathrm{C} 10-\mathrm{C} 9-\mathrm{C} 8$ & $122.55(15)$ & $\mathrm{C} 25-\mathrm{C} 24-\mathrm{H} 24$ & 120.0 \\
\hline $\mathrm{C} 14-\mathrm{C} 9-\mathrm{C} 8$ & $116.51(14)$ & $\mathrm{C} 23-\mathrm{C} 24-\mathrm{H} 24$ & 120.0 \\
\hline $\mathrm{C} 11-\mathrm{C} 10-\mathrm{C} 9$ & $119.71(16)$ & $\mathrm{C} 26-\mathrm{C} 25-\mathrm{C} 24$ & $120.80(16)$ \\
\hline $\mathrm{C} 11-\mathrm{C} 10-\mathrm{H} 10$ & 120.1 & $\mathrm{C} 26-\mathrm{C} 25-\mathrm{H} 25$ & 119.6 \\
\hline $\mathrm{C} 9-\mathrm{C} 10-\mathrm{H} 10$ & 120.1 & $\mathrm{C} 24-\mathrm{C} 25-\mathrm{H} 25$ & 119.6 \\
\hline $\mathrm{C} 10-\mathrm{C} 11-\mathrm{C} 12$ & $120.25(16)$ & $\mathrm{C} 25-\mathrm{C} 26-\mathrm{C} 27$ & $119.43(16)$ \\
\hline $\mathrm{C} 10-\mathrm{C} 11-\mathrm{H} 11$ & 119.9 & $\mathrm{C} 25-\mathrm{C} 26-\mathrm{H} 26$ & 120.3 \\
\hline $\mathrm{C} 12-\mathrm{C} 11-\mathrm{H} 11$ & 119.9 & $\mathrm{C} 27-\mathrm{C} 26-\mathrm{H} 26$ & 120.3 \\
\hline $\mathrm{C} 13-\mathrm{C} 12-\mathrm{C} 11$ & $120.41(16)$ & $\mathrm{C} 28-\mathrm{C} 27-\mathrm{C} 26$ & $120.56(16)$ \\
\hline $\mathrm{C} 13-\mathrm{C} 12-\mathrm{H} 12$ & 119.8 & $\mathrm{C} 28-\mathrm{C} 27-\mathrm{H} 27$ & 119.7 \\
\hline $\mathrm{C} 11-\mathrm{C} 12-\mathrm{H} 12$ & 119.8 & $\mathrm{C} 26-\mathrm{C} 27-\mathrm{H} 27$ & 119.7 \\
\hline $\mathrm{C} 12-\mathrm{C} 13-\mathrm{C} 14$ & $121.48(16)$ & $\mathrm{C} 27-\mathrm{C} 28-\mathrm{C} 23$ & $119.98(16)$ \\
\hline $\mathrm{C} 12-\mathrm{C} 13-\mathrm{H} 13$ & 119.3 & $\mathrm{C} 27-\mathrm{C} 28-\mathrm{H} 28$ & 120.0 \\
\hline $\mathrm{C} 14-\mathrm{C} 13-\mathrm{H} 13$ & 119.3 & $\mathrm{C} 23-\mathrm{C} 28-\mathrm{H} 28$ & 120.0 \\
\hline $\mathrm{O} 2-\mathrm{C} 1-\mathrm{C} 2-\mathrm{C} 3$ & $-6.4(2)$ & $\mathrm{C} 15-\mathrm{C} 16-\mathrm{C} 17-\mathrm{C} 18$ & $1.1(2)$ \\
\hline $\mathrm{O} 1-\mathrm{C} 1-\mathrm{C} 2-\mathrm{C} 3$ & $172.81(15)$ & $\mathrm{C} 16-\mathrm{C} 17-\mathrm{C} 18-\mathrm{C} 19$ & $-0.3(2)$ \\
\hline $\mathrm{O} 2-\mathrm{C} 1-\mathrm{C} 2-\mathrm{C} 7$ & $174.49(15)$ & $\mathrm{C} 17-\mathrm{C} 18-\mathrm{C} 19-\mathrm{C} 20$ & $-0.7(2)$ \\
\hline $\mathrm{O} 1-\mathrm{C} 1-\mathrm{C} 2-\mathrm{C} 7$ & $-6.2(2)$ & $\mathrm{C} 18-\mathrm{C} 19-\mathrm{C} 20-\mathrm{C} 15$ & $1.0(2)$ \\
\hline $\mathrm{C} 7-\mathrm{C} 2-\mathrm{C} 3-\mathrm{C} 4$ & $2.3(3)$ & $\mathrm{C} 18-\mathrm{C} 19-\mathrm{C} 20-\mathrm{C} 21$ & $-178.48(15)$ \\
\hline $\mathrm{C} 1-\mathrm{C} 2-\mathrm{C} 3-\mathrm{C} 4$ & $-176.81(16)$ & $\mathrm{C} 16-\mathrm{C} 15-\mathrm{C} 20-\mathrm{C} 19$ & $-0.2(2)$ \\
\hline $\mathrm{C} 2-\mathrm{C} 3-\mathrm{C} 4-\mathrm{C} 5$ & $-1.0(3)$ & $\mathrm{C} 14-\mathrm{C} 15-\mathrm{C} 20-\mathrm{C} 19$ & $-179.56(14)$ \\
\hline $\mathrm{C} 3-\mathrm{C} 4-\mathrm{C} 5-\mathrm{C} 6$ & $-1.2(3)$ & $\mathrm{C} 16-\mathrm{C} 15-\mathrm{C} 20-\mathrm{C} 21$ & $179.29(13)$ \\
\hline $\mathrm{C} 4-\mathrm{C} 5-\mathrm{C} 6-\mathrm{C} 7$ & $2.0(3)$ & $\mathrm{C} 14-\mathrm{C} 15-\mathrm{C} 20-\mathrm{C} 21$ & $-0.1(2)$ \\
\hline $\mathrm{C} 5-\mathrm{C} 6-\mathrm{C} 7-\mathrm{C} 2$ & $-0.7(2)$ & $\mathrm{N} 1-\mathrm{C} 8-\mathrm{C} 21-\mathrm{N} 2$ & $-0.15(16)$ \\
\hline $\mathrm{C} 3-\mathrm{C} 2-\mathrm{C} 7-\mathrm{C} 6$ & $-1.4(2)$ & $\mathrm{C} 9-\mathrm{C} 8-\mathrm{C} 21-\mathrm{N} 2$ & $179.75(13)$ \\
\hline $\mathrm{C} 1-\mathrm{C} 2-\mathrm{C} 7-\mathrm{C} 6$ & $177.64(14)$ & $\mathrm{N} 1-\mathrm{C} 8-\mathrm{C} 21-\mathrm{C} 20$ & $178.83(13)$ \\
\hline $\mathrm{C} 22-\mathrm{N} 1-\mathrm{C} 8-\mathrm{C} 21$ & $-0.49(16)$ & $\mathrm{C} 9-\mathrm{C} 8-\mathrm{C} 21-\mathrm{C} 20$ & $-1.3(2)$ \\
\hline $\mathrm{C} 22-\mathrm{N} 1-\mathrm{C} 8-\mathrm{C} 9$ & $179.62(15)$ & $\mathrm{C} 22-\mathrm{N} 2-\mathrm{C} 21-\mathrm{C} 8$ & $0.74(16)$ \\
\hline $\mathrm{C} 21-\mathrm{C} 8-\mathrm{C} 9-\mathrm{C} 10$ & $-178.91(14)$ & $\mathrm{C} 22-\mathrm{N} 2-\mathrm{C} 21-\mathrm{C} 20$ & $-178.15(15)$ \\
\hline $\mathrm{N} 1-\mathrm{C} 8-\mathrm{C} 9-\mathrm{C} 10$ & $1.0(2)$ & $\mathrm{C} 19-\mathrm{C} 20-\mathrm{C} 21-\mathrm{C} 8$ & $-179.40(15)$ \\
\hline $\mathrm{C} 21-\mathrm{C} 8-\mathrm{C} 9-\mathrm{C} 14$ & $0.3(2)$ & $\mathrm{C} 15-\mathrm{C} 20-\mathrm{C} 21-\mathrm{C} 8$ & $1.1(2)$ \\
\hline
\end{tabular}




$\begin{array}{llll}\mathrm{N} 1-\mathrm{C} 8-\mathrm{C} 9-\mathrm{C} 14 & -179.82(14) & \mathrm{C} 19-\mathrm{C} 20-\mathrm{C} 21-\mathrm{N} 2 & -0.7(2) \\ \mathrm{C} 14-\mathrm{C} 9-\mathrm{C} 10-\mathrm{C} 11 & -1.0(2) & \mathrm{C} 15-\mathrm{C} 20-\mathrm{C} 21-\mathrm{N} 2 & 179.84(14) \\ \mathrm{C} 8-\mathrm{C} 9-\mathrm{C} 10-\mathrm{C} 11 & 178.22(14) & \mathrm{C} 8-\mathrm{N} 1-\mathrm{C} 22-\mathrm{N} 2 & 0.96(16) \\ \mathrm{C} 9-\mathrm{C} 10-\mathrm{C} 11-\mathrm{C} 12 & 0.3(2) & \mathrm{C} 8-\mathrm{N} 1-\mathrm{C} 22-\mathrm{C} 23 & -176.97(14) \\ \mathrm{C} 10-\mathrm{C} 11-\mathrm{C} 12-\mathrm{C} 13 & 0.6(2) & \mathrm{C} 21-\mathrm{N} 2-\mathrm{C} 22-\mathrm{N} 1 & -1.05(16) \\ \mathrm{C} 11-\mathrm{C} 12-\mathrm{C} 13-\mathrm{C} 14 & -0.8(2) & \mathrm{C} 21-\mathrm{N} 2-\mathrm{C} 22-\mathrm{C} 23 & 176.90(14) \\ \mathrm{C} 12-\mathrm{C} 13-\mathrm{C} 14-\mathrm{C} 9 & 0.2(2) & \mathrm{N} 1-\mathrm{C} 22-\mathrm{C} 23-\mathrm{C} 24 & 175.89(14) \\ \mathrm{C} 12-\mathrm{C} 13-\mathrm{C} 14-\mathrm{C} 15 & -179.05(15) & \mathrm{N} 2-\mathrm{C} 22-\mathrm{C} 23-\mathrm{C} 24 & -1.7(2) \\ \mathrm{C} 10-\mathrm{C} 9-\mathrm{C} 14-\mathrm{C} 13 & 0.7(2) & \mathrm{N} 1-\mathrm{C} 22-\mathrm{C} 23-\mathrm{C} 28 & -3.0(2) \\ \mathrm{C} 8-\mathrm{C} 9-\mathrm{C} 14-\mathrm{C} 13 & -178.51(13) & \mathrm{N} 2-\mathrm{C} 22-\mathrm{C} 23-\mathrm{C} 28 & 179.44(14) \\ \mathrm{C} 10-\mathrm{C} 9-\mathrm{C} 14-\mathrm{C} 15 & 179.95(14) & \mathrm{C} 28-\mathrm{C} 23-\mathrm{C} 24-\mathrm{C} 25 & -1.2(2) \\ \mathrm{C} 8-\mathrm{C} 9-\mathrm{C} 14-\mathrm{C} 15 & 0.7(2) & \mathrm{C} 22-\mathrm{C} 23-\mathrm{C} 24-\mathrm{C} 25 & 179.97(14) \\ \mathrm{C} 13-\mathrm{C} 14-\mathrm{C} 15-\mathrm{C} 16 & -1.0(2) & \mathrm{C} 23-\mathrm{C} 24-\mathrm{C} 25-\mathrm{C} 26 & -0.1(2) \\ \mathrm{C} 9-\mathrm{C} 14-\mathrm{C} 15-\mathrm{C} 16 & 179.84(14) & \mathrm{C} 24-\mathrm{C} 25-\mathrm{C} 26-\mathrm{C} 27 & 1.0(2) \\ \mathrm{C} 13-\mathrm{C} 14-\mathrm{C} 15-\mathrm{C} 20 & 178.35(14) & \mathrm{C} 25-\mathrm{C} 26-\mathrm{C} 27-\mathrm{C} 28 & -0.6(2) \\ \mathrm{C} 9-\mathrm{C} 14-\mathrm{C} 15-\mathrm{C} 20 & -0.8(2) & \mathrm{C} 26-\mathrm{C} 27-\mathrm{C} 28-\mathrm{C} 23 & -0.7(2) \\ \mathrm{C} 20-\mathrm{C} 15-\mathrm{C} 16-\mathrm{C} 17 & -0.8(2) & \mathrm{C} 24-\mathrm{C} 23-\mathrm{C} 28-\mathrm{C} 27 & 1.5(2) \\ \mathrm{C} 14-\mathrm{C} 15-\mathrm{C} 16-\mathrm{C} 17 & 178.53(15) & \mathrm{C} 22-\mathrm{C} 23-\mathrm{C} 28-\mathrm{C} 27 & -179.60(14) \\ \end{array}$

Hydrogen-bond geometry $\left(A,{ }^{\circ}\right)$

$\mathrm{Cg} 4$ is the centroid of the $\mathrm{C} 15-\mathrm{C} 20$ benzene ring.

\begin{tabular}{lllll}
\hline$D-\mathrm{H} \cdots A$ & $D-\mathrm{H}$ & $\mathrm{H} \cdots A$ & $D \cdots A$ & $D-\mathrm{H} \cdots A$ \\
\hline $\mathrm{N} 1-\mathrm{H} 1 \cdots \mathrm{O} 1$ & 0.86 & 1.77 & $2.6159(17)$ & 168 \\
$\mathrm{~N} 2-\mathrm{H} 2 \cdots \mathrm{O} 2^{\mathrm{i}}$ & 0.86 & 1.83 & $2.6523(16)$ & 158 \\
$\mathrm{C} 7-\mathrm{H} 7 \cdots C g 4^{\mathrm{ii}}$ & 0.93 & 2.79 & $3.585(2)$ & 145 \\
$\mathrm{C} 10-\mathrm{H} 10 \cdots \mathrm{O} 1$ & 0.93 & 2.40 & $3.265(2)$ & 155 \\
$\mathrm{C} 19-\mathrm{H} 19 \cdots \mathrm{O} 2^{\mathrm{i}}$ & 0.93 & 2.54 & $3.372(2)$ & 150 \\
$\mathrm{C} 24-\mathrm{H} 24 \cdots \mathrm{O} 2^{\mathrm{i}}$ & 0.93 & 2.50 & $3.343(2)$ & 152 \\
$\mathrm{C} 28-\mathrm{H} 28 \cdots \mathrm{O} 1$ & 0.93 & 2.48 & $3.365(2)$ & 159
\end{tabular}

Symmetry codes: (i) $-x+1,-y+1,-z+1$; (ii) $-x,-y+1,-z+1$. 